\title{
Galactic Wolf-Rayet stars in the mid-infrared
}

\author{
John D. Smith and Jim R. Houck \\ Cornell University Department of Astronomy, Ithaca, NY 14853
}

\begin{abstract}
We present the results of observations of a sample of Galactic WR stars with SCORE, the SIRTF Cornell Echelle spectrograph. Spectra with resolution $R \simeq 600$ and wavelength coverage from $7.5-15 \mu \mathrm{m}$ were obtained for a variety of WR subtypes.
\end{abstract}

\section{Observations}

The mid-infrared $N$-band spectra of 20 galactic Wolf-Rayet stars were obtained with SCORE, the SIRTF Cornell Echelle Spectrograph, on the Palomar Hale $5 \mathrm{~m}$ telescope. SCORE is a crossed-echelle spectrograph which captures the entire 7.5$15 \mu \mathrm{m}$ window in a single exposure. SCORE is a prototype for the SIRTF-IRS short-High spectrographic module (Smith et al. 1998; Van Cleve et al. 1998).

Among the nitrogen WR stars observed were one WN5, four WN6, one WN7/Of, three WN8 stars, and one Ofpe/WN9 object. The carbon WR star

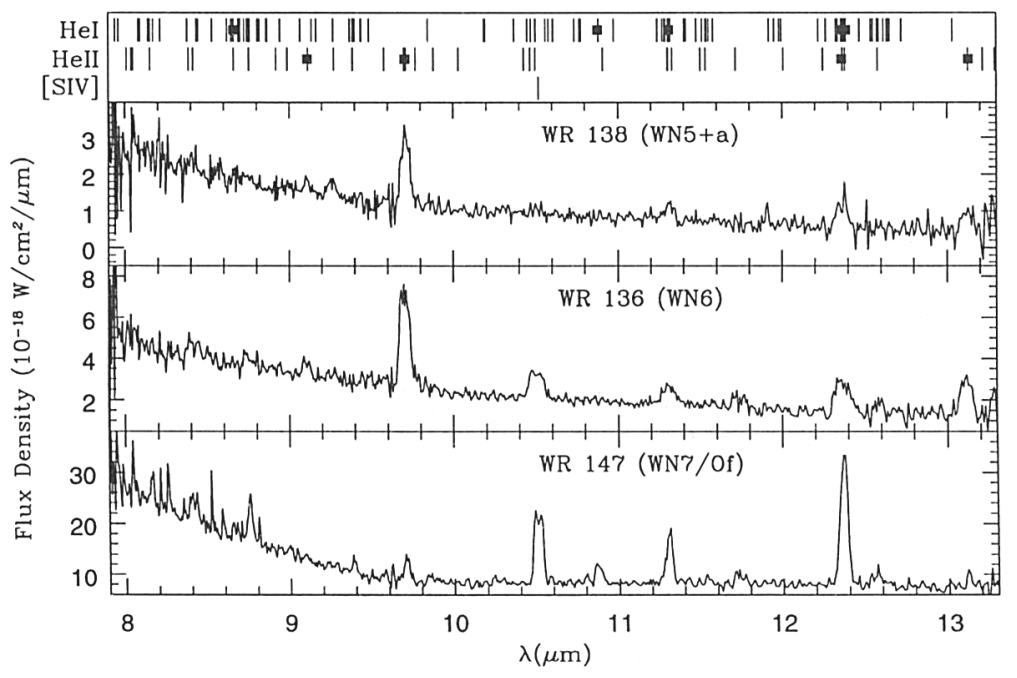

Figure 1. A sample of the WN stars observed. Listed He lines with a square mark are taken from a UKIRT catalog of observed lines. 


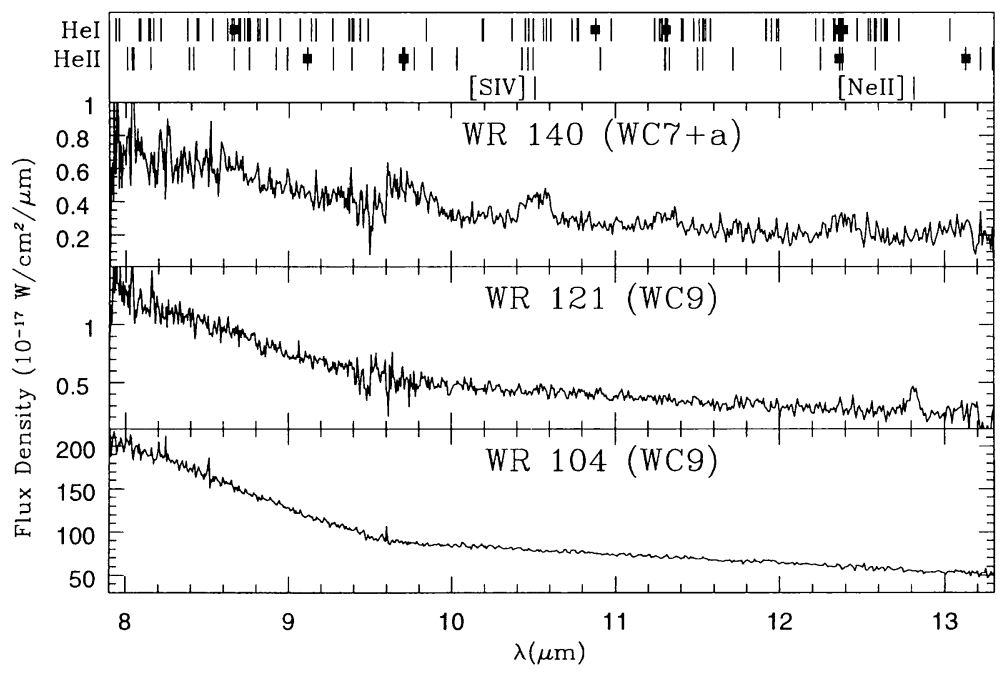

Figure 2. A sample of the WC stars observed, including both dusty and line-rich examples.

observations consisted of one WC5, three WC7, one WC8, and five WC9 stars. All data were taken between December, 1997 and July, 1998.

\section{Summary}

Most WN spectra contain recombination lines of He, with occasional fine structure lines of [NeII] and [SIV]. The observed WN stars exhibit significant variation of line strengths and widths both within and between individual subclasses. Warm dust emission with a break in spectral slope at $\sim 9.7 \mu \mathrm{m}$ is evident in most of the late WC stars. However, the same break, normally attributed to silicate absorption, also occurs in several mid- to late-type WN's, though less pronounced than in the WC's. This is a possible indication either that the absorbing silicates are predominantly interstellar rather than circumstellar, or that the abrupt change in slope is due to a fundamental (subtype independent) property of Wolf-Rayet winds. Sample spectra are shown in Figs. 1 and 2. Note the $9.7 \mu \mathrm{m}$ break in the spectrum of WR 147 (WN), observed before by ISO-sWs (van der Hucht et al. 1996). Also note the presence of [NeII] in WR 121 (WC9), as seen before in WR 11 (WC8) (van der Hucht et al. 1996).

\section{References}

van der Hucht, K.A., Morris, P.W., Williams, P.M., et al. 1996, A\&A 315, L193

Smith, J.D. et al. 1998, Proc. SPIE, 3354, 798

Van Cleve, J., Gull, G.E., Rinehart, S.A., Smith, J.D., Wilson, J.C, Houck, J.R., Brown, R., Blalock, W. 1998, PASP 110, 1479 